\title{
Assessment of Continuous Oil and Gas Resources in the Neuquén Basin Province, Argentina, 2016
}

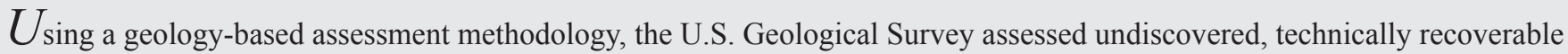
mean continuous resources of 14.4 billion barrels of oil and 38 trillion cubic feet of gas in the Neuquén Basin Province, Argentina.

\section{Introduction}

The U.S. Geological Survey (USGS) assessed the potential for undiscovered, technically recoverable continuous (unconventional) oil and gas resources within the Neuquén Basin Province, Argentina (figs. 1-3). As defined by the USGS, the province encompasses the basin (or "embayment"), the platform in the eastern part of the province, and the fold belt in the western part of the province. This study estimated potential volumes of undiscovered continuous oil and gas in three petroleum source rocks that occur in the basin and in the fold belt: the Jurassic Los Molles Formation, the Jurassic-Early Cretaceous Vaca Muerta Formation, and the Early Cretaceous Agrio Formation. The USGS defined a Los Molles Total Petroleum System (TPS), a Vaca Muerta TPS, and an Agrio TPS, following similar published definitions of these systems (Urien and Zambrano, 1994; Legarreta and Villar, 2015). Twelve continuous resource assessment units (AUs) were defined within these three TPSs (table 1).

\section{Geologic Model for Assessment}

Three main assumptions underlie the geologic model that forms the foundation of this assessment. First, some portion of the oil and gas generated in each of the three source rocks was retained within each source rock interval and constitutes potentially recoverable oil or gas. Second, multiple phases of structural deformation in the fold belt negatively affected the volumes of oil or gas retained in source rocks compared to those retained in the basin or embayment area. Third, the assessment was based mainly (but not entirely) on vertical wells, which affects the input data for the USGS assessment methodology (for example, well drainage areas, estimated ultimate recoveries, and success ratios). Given the geologic model, the definition of AUs was based on (1) whether the source rock resides within the fold belt or embayment, (2) the level of thermal maturation, (3) the percent of total organic

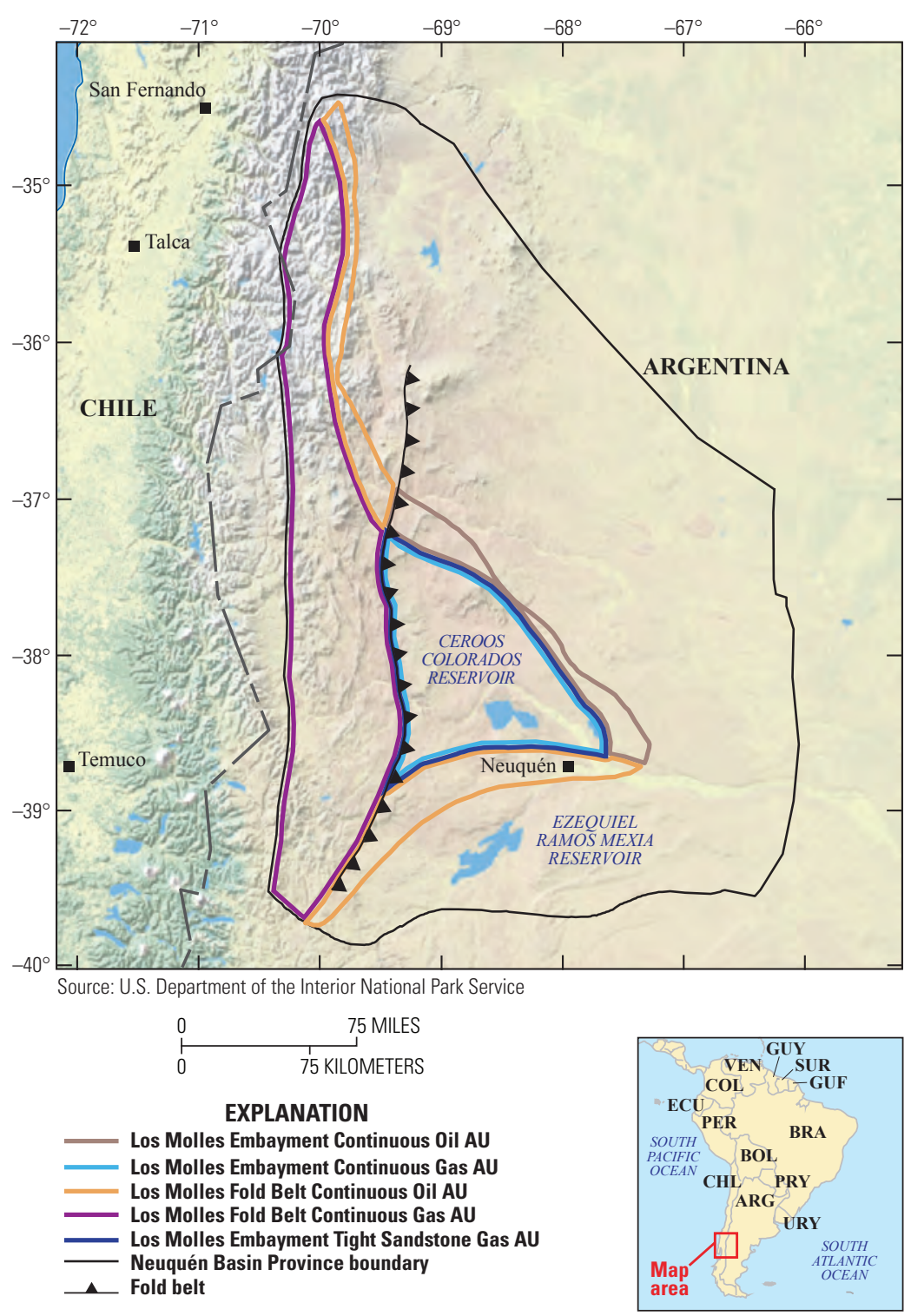

Figure 1. Location of the five assessment units (AUs) in the Los Molles Total Petroleum System of the Neuquén Basin Province, Argentina.

carbon, and (4) the thickness of viable source rock. Source rock data for the Los Molles TPS, Vaca Muerta TPS, and Agrio TPS from published sources are summarized in table 2 (Legarreta and others, 2005; Tyson and others, 2005; Martinez and others, 2008; Schmidt and others, 2013; 


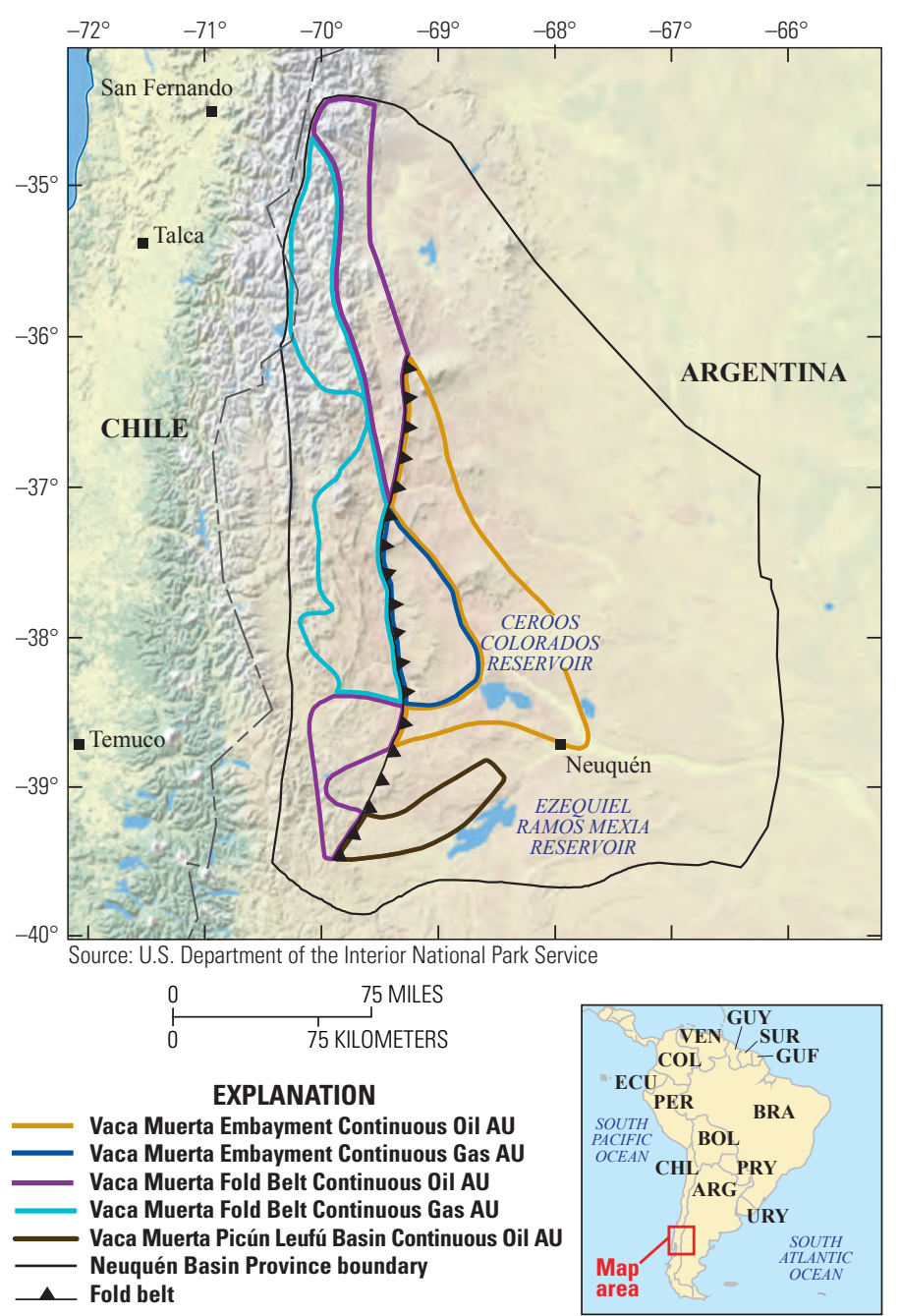

Figure 2. Location of the five assessment units (AUs) in the Vaca Muerta Total Petroleum System of the Neuquén Basin Province, Argentina.

Barredo and Stinco, 2014; Legarreta and Villar, 2015; Licitra and others, 2015; Rimedio and others, 2015). The lower limits of thickness of effective source rock (15 meters), total organic carbon ( 2 weight percent), and hydrogen index (200 milligrams hydrocarbon per gram of total organic carbon) are the thresholds used by the USGS to define a viable continuous accumulation (table 2).

Key assessment input data for 12 AUs are summarized in table 1. Data for estimated ultimate recovery, well drainage area, and success ratios were based on U.S. analogs for continuous oil and gas reservoirs.

\section{Undiscovered Resources Summary}

The USGS assessed potential undiscovered, technically recoverable continuous resources in the Neuquén Basin Province, Argentina (table 3). The USGS estimated mean totals of 14,370 million barrels of continuous oil (MMBO), or 14.4 billion barrels of oil, with an F95-F5 range from 4,139 to 27,913 MMBO; 37,966 billion cubic feet of continuous gas (BCFG), or 38 trillion cubic feet of continuous gas, with an F95-F5 range from 10,382 to 76,102 BCFG; and 482 million barrels of natural gas liquids (MMBNGL) with an F95-F5 range from 113 to 1,055 MMBNGL (table 3).

In the Los Molles TPS, the estimated mean totals for continuous oil and gas resources are $847 \mathrm{MMBO}$ with an F95-F5 range from 220 to 1,775 MMBO, 18,137 BCFG with an F95-F5 range from 4,993 to 36,000 BCFG, and 184 MMBNGL with an F95-F5 range from 44 to 400 MMBNGL. The Vaca Muerta TPS has estimated mean totals for continuous oil and gas resources of 12,848 MMBO with an F95-F5 range from 3,750 to 24,696 MMBO, 19,155 BCFG with an F95-F5 range from 5,232 to 38,597 BCFG, and 287 MMBNGL with an F95-F5 range from 67 to 630 MMBNGL. In the Agrio TPS, the estimated mean totals for continuous oil and gas resources are $675 \mathrm{MMBO}$ with an F95-F5 range from 169 to 1,442 MMBO, 674 BCFG with an F95-F5 range from 157 to 1,505 BCFG, and 11 MMBNGL with an F95-F5 range from 2 to 25 MMBNGL.

Most of the continuous oil resources (12,848 MMBO or 89 percent) are estimated to be in three assessment units of the Vaca Muerta TPS. Half of the mean continuous gas resources $(19,155 \mathrm{BCFG}$ or 50 percent) is in the Vaca Muerta TPS, and 48 percent $(18,137 \mathrm{BCFG})$ is in the Los Molles TPS.

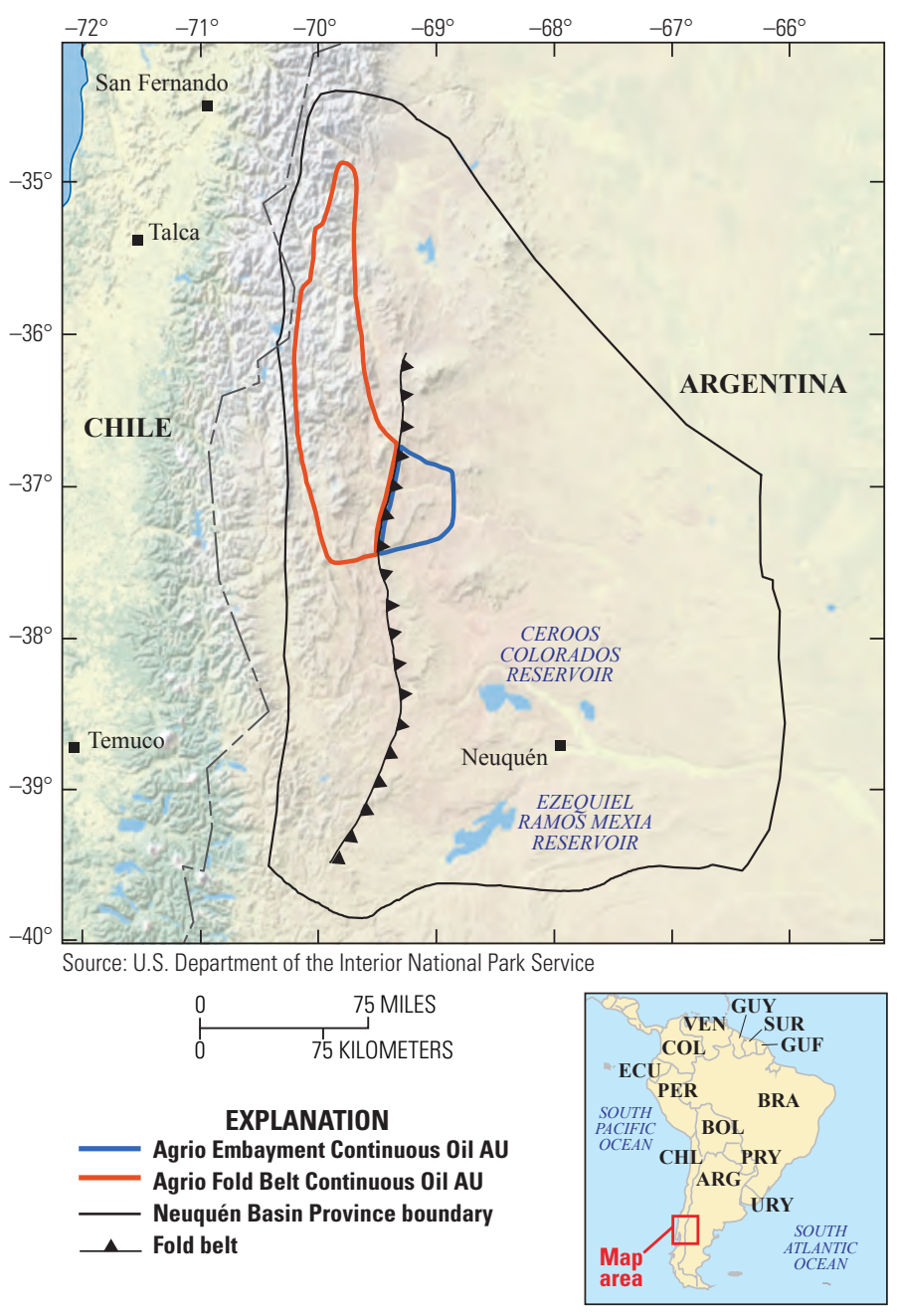

Figure 3. Location of the two assessment units (AUs) in the Agrio Total Petroleum System of the Neuquén Basin Province, Argentina. 
Table 1. Key assessment input data for 12 continuous assessment units in the Neuquén Basin Province, Argentina.

[AU, assessment unit; \%, percent; EUR, estimated ultimate recovery per well; MMBO, million barrels of oil; BCFG, billion cubic feet of gas. EUR, well drainage area, and well success ratios are from U.S. shale-gas, tight-gas, and shale-oil analogs. The EUR input includes the minimum, median, maximum, and calculated mean. Shading indicates not applicable]

\begin{tabular}{|c|c|c|c|c|c|c|c|c|}
\hline \multirow{2}{*}{ Assessment input data } & \multicolumn{4}{|c|}{ Los Molles Embayment Continuous Oil AU } & \multicolumn{4}{|c|}{ Los Molles Embayment Continuous Gas AU } \\
\hline & Minimum & Mode & Maximum & Calculated mean & Minimum & Mode & Maximum & Calculated mean \\
\hline Potential production area of AU (acres) & 2,400 & 480,000 & 960,000 & 480,800 & 4,800 & $1,556,000$ & $3,112,000$ & $1,557,600$ \\
\hline Average drainage area of wells (acres) & 20 & 60 & 120 & 67 & 80 & 120 & 160 & 120 \\
\hline Success ratios (\%) & 50 & 70 & 90 & 70 & 50 & 70 & 90 & 70 \\
\hline Average EUR (MMBO, oil; BCFG, gas) & 0.08 & 0.1 & 0.3 & 0.111 & 0.2 & 0.4 & 1.0 & 0.431 \\
\hline AU probability & 1.0 & & & & 1.0 & & & \\
\hline \multirow{2}{*}{ Assessment input data } & \multicolumn{4}{|c|}{ Los Molles Fold Belt Continuous Oil AU } & \multicolumn{4}{|c|}{ Los Molles Fold Belt Continuous Gas AU } \\
\hline & Minimum & Mode & Maximum & Calculated mean & Minimum & Mode & Maximum & Calculated mean \\
\hline Potential production area of AU (acres) & 600 & 717,000 & $2,391,000$ & $1,036,200$ & 3,000 & $2,407,000$ & $8,670,000$ & $3,693,333$ \\
\hline Average drainage area of wells (acres) & 40 & 80 & 120 & 80 & 80 & 120 & 160 & 120 \\
\hline Success ratios (\%) & 10 & 30 & 50 & 30 & 10 & 30 & 50 & 30 \\
\hline Average EUR (MMBO, oil; BCFG, gas) & 0.03 & 0.06 & 0.1 & 0.062 & 0.2 & 0.35 & 0.8 & 0.373 \\
\hline AU probability & 1.0 & & & & 1.0 & & & \\
\hline \multirow{2}{*}{ Assessment input data } & \multicolumn{4}{|c|}{ Los Molles Embayment Tight Sandstone Gas AU } & \multicolumn{4}{|c|}{ Vaca Muerta Embayment Continuous Oil AU } \\
\hline & Minimum & Mode & Maximum & Calculated mean & Minimum & Mode & Maximum & Calculated mean \\
\hline Potential production area of AU (acres) & 9,600 & $1,800,000$ & $3,112,000$ & $1,640,533$ & 3,000 & $2,407,000$ & $3,438,000$ & $1,949,333$ \\
\hline Average drainage area of wells (acres) & 40 & 80 & 120 & 80 & 20 & 60 & 120 & 67 \\
\hline Success ratios (\%) & 65 & 75 & 85 & 75 & 75 & 85 & 95 & 85 \\
\hline Average EUR (BCFG, gas; MMBO, oil) & 0.2 & 0.6 & 1.4 & 0.637 & 0.2 & 0.4 & 0.7 & 0.412 \\
\hline AU probability & 1.0 & & & & 1.0 & & & \\
\hline \multirow{2}{*}{ Assessment input data } & \multicolumn{4}{|c|}{ Vaca Muerta Embayment Continuous Gas AU } & \multicolumn{4}{|c|}{ Vaca Muerta Fold Belt Continuous Oil AU } \\
\hline & Minimum & Mode & Maximum & Calculated mean & Minimum & Mode & Maximum & Calculated mean \\
\hline Potential production area of AU (acres) & 6,000 & 928,000 & $1,325,000$ & 753,000 & 600 & $1,005,000$ & $3,350,000$ & $1,451,867$ \\
\hline Average drainage area of wells (acres) & 80 & 120 & 160 & 120 & 40 & 80 & 120 & 80 \\
\hline Success ratios (\%) & 75 & 85 & 95 & 85 & 10 & 30 & 50 & 30 \\
\hline Average EUR (BCFG, gas; MMBO, oil) & 0.2 & 0.8 & 2.0 & 0.856 & 0.03 & 0.06 & 0.10 & 0.062 \\
\hline AU probability & 1.0 & & & & 1.0 & & & \\
\hline \multirow{2}{*}{ Assessment input data } & \multicolumn{4}{|c|}{ Vaca Muerta Fold Belt Continuous Gas AU } & \multicolumn{4}{|c|}{ Vaca Muerta Picún Leufú Basin Continuous Oil AU } \\
\hline & Minimum & Mode & Maximum & Calculated mean & Minimum & Mode & Maximum & Calculated mean \\
\hline Potential production area of AU (acres) & 1,200 & $1,332,000$ & $4,439,000$ & $1,934,067$ & 3,000 & 690,000 & 986,000 & 559,667 \\
\hline Average drainage area of wells (acres) & 80 & 120 & 160 & 120 & 20 & 60 & 120 & 67 \\
\hline Success ratios (\%) & 10 & 30 & 50 & 30 & 70 & 80 & 90 & 80 \\
\hline Average EUR (BCFG, gas; MMBO, oil) & 0.2 & 0.35 & 0.8 & 0.373 & 0.1 & 0.2 & 0.35 & 0.206 \\
\hline AU probability & 1.0 & & & & 1.0 & & & \\
\hline \multirow{2}{*}{ Assessment input data } & \multicolumn{4}{|c|}{ Agrio Embayment Continuous Oil AU } & \multicolumn{4}{|c|}{ Agrio Fold Belt Continuous Oil AU } \\
\hline & Minimum & Mode & Maximum & Calculated mean & Minimum & Mode & Maximum & Calculated mean \\
\hline Potential production area of AU (acres) & 2,400 & 268,000 & 535,000 & 268,467 & 600 & $1,085,000$ & $3,615,000$ & $1,566,867$ \\
\hline Average drainage area of wells (acres) & 20 & 60 & 120 & 67 & 40 & 80 & 120 & 80 \\
\hline Success ratios (\%) & 50 & 70 & 90 & 70 & 10 & 30 & 50 & 30 \\
\hline Average EUR (MMBO) & 0.06 & 0.09 & 0.3 & 0.102 & 0.03 & 0.06 & 0.1 & 0.062 \\
\hline AU probability & 1.0 & & & & 1.0 & & & \\
\hline
\end{tabular}

\section{References Cited}

Barredo, S.P., and Stinco, L.P., 2014, Unconventional reservoir geology of the Neuquén Basin, Argentina, in Society of Petroleum Engineers, SPE Annual Conference and Exhibition, Amsterdam, Netherlands, October 27-29, 2014, Proceedings: Society of Petroleum Engineers, Paper SPE-170905-MS, 11 p., accessed September 30, 2016, at https://www.onepetro.org/conference-paper/SPE-170905-MS.

Legarreta, Leonardo, Cruz, C.E., Vergani, Gustavo, Laffitte, G.A., and Villar, H.J., 2005, Petroleum mass-balance of the Neuquén Basin, Argentina-A comparative assessment of the productive districts and non-productive trends: American Association of Petroleum Geologists, Search and Discovery Article No. 10080, 7 p., accessed September 30, 2016 , at http://www.searchanddiscovery.com/pdfz/documents/2005/legarreta/images/legarreta.pdf.html.

Legarreta, Leonardo, and Villar, J.J., 2015, The Vaca Muerta Formation (Late Jurassic-Early Cretaceous), Neuquén Basin, Argentina-Sequences, facies, and source rock characteristics, in Unconventional Resources Technology Conference, San Antonio, Texas, July 20-22, 2015, Proceedings: Unconventional Resources Technology Conference, Paper URTeC:2170906, 15 p., accessed September 30, 2016, at http://www.geolabsur.com/Biblioteca/Legarreta_Villar_2015_VM_Urtec.pdf.

Licitra, Diego; Lovrincevich, Eliana; Vittore, Franco; Quiroga, Juan; Oviedo, Pablo; Montoya, Veronica; Shannon, Coleen; and Monti, Luciano, 2015, Sweet spots in Vaca Muerta Integration of subsurface and production data in Loma Campana shale development, Argentina, in Unconventional Resources Technology Conference, San Antonio, Texas, July 20-22, 2015, Proceedings: Unconventional Resources Technology Conference, Paper URTeC:2153944, 18 p., accessed September 30, 2016, at https://www.onepetro.org/ conference-paper/SPE-178563-MS.

Martínez, M.A., Prámparo, M.B., Quattrocchio, M.E., and Zavala, C.A., 2008, Depositional environments and hydrocarbon potential of the Middle Jurassic Los Molles Formation, Neuquén Basin, Argentina-Palynofacies and organic geochemical data: Andean Geology (formerly known as Revista Geológica de Chile), v. 35, no. 2, p. $235-247$.

Rimedio, Milena; Shannon, Coleen; Monti, Luciano; Lerza, Alejandro; Roberts, Matthew; and Quiroga, Juan, 2015, Interference behavior analysis in Vaca Muerta shale oil development, Loma Campana Field, Argentina, in Unconventional Resources Technology Conference, San Antonio, Texas, July 20-22, 2015, Proceedings: Unconventional Resources Technology Conference, MS/URTeC:2154859, 14 p., accessed September 30, 2016, at https://www.onepetro.org/conference-paper/SPE-178620-MS.

Schmidt, N.G., Alonso, J.C., and Giusiano, Adolfo, 2013, Assessment of Vaca Muerta Formation shale oil—Production decline-curve analysis, in Workshop on Unconventional Reservoirs, Ministry of Energy, Neuquén Province, Argentina, April 17, 2013: Workshop on Unconventional Reservoirs, 31 p., accessed September 30, 2016 , at http://www.energianeuquen.gov.ar/cms/files/contenido/70/4Evaluacion\%20Shale\%20IAPG\%20abril2013.pdf.

Tyson, R.V., Esherwood, Philip, and Pattison, K.A., 2005, Organic facies in the Valanginian-mid-Hauterivian interval of the Agrio Formation (Chos Malal area, Neuquén, Argentina) - Local significance and global context, in Veiga, G.D., Spalletti, L.A., Howell, J.A., and Schwarz, E., eds., The Neuquén Basin, Argentina-A case study in sequence stratigraphy and basin dynamics: Geological Society of London, Special Publication No. 252, p. 251-266.

Urien, C.M., and Zambrano, J.J., 1994, Petroleum systems in the Neuquén Basin, Argentina, in Magoon, L.B., and Dow, W.G., eds., The petroleum system-From source to trap: American Association of Petroleum Geologists Memoir No. 60, p. 513-534. 
Table 2. Summary of source rock data for three total petroleum systems in the Neuquén Basin Province, Argentina.

[TPS, total petroleum system; m, meters; wt \%, weight percent; $\% \mathrm{R}_{0}$, percent vitrinite reflectance; $\mathrm{mg} \mathrm{HC} / \mathrm{g}$ TOC, milligrams of hydrocarbon per gram of total organic carbon]

\begin{tabular}{|l|c|c|c|}
\hline & $\begin{array}{c}\text { Los Molles } \\
\text { TPS }\end{array}$ & $\begin{array}{c}\text { Vaca Muerta } \\
\text { TPS }\end{array}$ & $\begin{array}{c}\text { Agrio } \\
\text { TPS }\end{array}$ \\
\hline Maximum formation thickness $(\mathrm{m})$ & 2,100 & 1,200 & 1,500 \\
\hline Thickness of effective source rock $(\mathrm{m})$ & $15-800$ & $15-450$ & $15-400$ \\
\hline Total organic carbon $(\mathrm{wt} \%)$ & $2-5$ & $2-12$ & $2-7$ \\
\hline Maximum thermal maturation $\left(\% \mathrm{R}_{\mathrm{o}}\right)$ & 3 & 2 & 3.6 \\
\hline Hydrogen index $(\mathrm{mg} \mathrm{HC} / \mathrm{g}$ TOC) & $200-500$ & $200-800$ & $200-550$ \\
\hline Kerogen type & III/II & I/II/IIS & II/III \\
\hline
\end{tabular}

Table 3. Assessment results for 12 assessment units in the Neuquén Basin Province, Argentina.

[MMBO, million barrels of oil; BCFG, billions of cubic feet of gas; NGL, natural gas liquids; MMBNGL, million barrels of natural gas liquids. Results shown are fully risked estimates. For gas accumulations, all liquids are included as natural gas liquids. F95 represents a 95-percent chance of at least the tabulated amount being present; other fractiles are defined similarly. Fractiles are additive under the assumption of perfect positive correlation. Shading indicates not applicable]

\begin{tabular}{|c|c|c|c|c|c|c|c|c|c|c|c|c|c|c|}
\hline \multirow{3}{*}{$\begin{array}{l}\text { Total petroleum systems } \\
\text { and assessment units (AUs) }\end{array}$} & \multirow{3}{*}{$\begin{array}{l}\text { AU } \\
\text { prob- } \\
\text { ability } \\
\end{array}$} & \multirow{3}{*}{$\begin{array}{c}\text { Accu- } \\
\text { mulation } \\
\text { type }\end{array}$} & \multicolumn{12}{|c|}{ Total undiscovered resources } \\
\hline & & & \multicolumn{4}{|c|}{ Oil (MMBO) } & \multicolumn{4}{|c|}{ Gas (BCFG) } & \multicolumn{4}{|c|}{ NGL (MMBNGL) } \\
\hline & & & $\mathrm{F95}$ & F50 & F5 & Mean & F95 & F50 & $\mathrm{F5}$ & Mean & F95 & F50 & F5 & Mean \\
\hline \multicolumn{15}{|c|}{ Los Molles Total Petroleum System } \\
\hline $\begin{array}{c}\text { Los Molles Embayment } \\
\text { Continuous Oil AU }\end{array}$ & 1.0 & Oil & 163 & 544 & 1,236 & 601 & 151 & 528 & 1,298 & 601 & 2 & 8 & 21 & 9 \\
\hline $\begin{array}{c}\text { Los Molles Embayment } \\
\text { Continuous Gas AU }\end{array}$ & 1.0 & Gas & & & & & 1,116 & 3,644 & 7,631 & 3,913 & 15 & 52 & 126 & 59 \\
\hline \begin{tabular}{|c|} 
Los Molles Fold Belt \\
Continuous Oil AU \\
\end{tabular} & 1.0 & Oil & 57 & 215 & 539 & 246 & 54 & 210 & 562 & 246 & 1 & 3 & 9 & 4 \\
\hline $\begin{array}{r}\text { Los Molles Fold Belt } \\
\text { Continuous Gas AU } \\
\end{array}$ & 1.0 & Gas & & & & & 779 & 2,982 & 7,665 & 3,450 & 10 & 43 & 124 & 52 \\
\hline $\begin{array}{l}\text { Los Molles Embayment } \\
\text { Tight Sandstone Gas AU }\end{array}$ & 1.0 & Gas & & & & & 2,893 & 9,374 & 18,844 & 9,927 & 16 & 55 & 120 & 60 \\
\hline Total resources & & & 220 & 759 & 1,775 & 847 & 4,993 & 16,738 & 36,000 & 18,137 & 44 & 161 & 400 & 184 \\
\hline \multicolumn{15}{|c|}{ Vaca Muerta Total Petroleum System } \\
\hline $\begin{array}{c}\text { Vaca Muerta Embayment } \\
\text { Continuous Oil AU }\end{array}$ & 1.0 & Oil & 3,228 & 10,365 & 21,092 & 11,008 & 3,004 & 10,057 & 22,253 & 11,007 & 39 & 144 & 363 & 165 \\
\hline $\begin{array}{c}\text { Vaca Muerta Embayment } \\
\text { Continuous Gas AU }\end{array}$ & 1.0 & Gas & & & & & 1,329 & 4,261 & 8,587 & 4,512 & 17 & 61 & 141 & 68 \\
\hline $\begin{array}{c}\text { Vaca Muerta Fold Belt } \\
\text { Continuous Oil AU }\end{array}$ & 1.0 & Oil & 79 & 303 & 753 & 345 & 74 & 294 & 785 & 344 & 1 & 4 & 13 & 5 \\
\hline $\begin{array}{r}\text { Vaca Muerta Fold Belt } \\
\text { Continuous Gas AU }\end{array}$ & 1.0 & Gas & & & & & 412 & 1,562 & 3,972 & 1,798 & 5 & 23 & 64 & 27 \\
\hline \begin{tabular}{|c|} 
Vaca Muerta Picún Leufú \\
Basin Continuous Oil AU \\
\end{tabular} & 1.0 & Oil & 443 & 1,415 & 2,851 & 1,495 & 413 & 1,370 & 3,000 & 1,494 & 5 & 20 & 49 & 22 \\
\hline Total resources & & & 3,750 & 12,083 & 24,696 & 12,848 & 5,232 & 17,544 & $\mathbf{3 8 , 5 9 7}$ & 19,155 & 67 & 252 & 630 & 287 \\
\hline \multicolumn{15}{|c|}{ Agrio Total Petroleum System } \\
\hline $\begin{array}{l}\text { Agrio Embayment } \\
\text { Continuous Oil AU } \\
\end{array}$ & 1.0 & Oil & 82 & 272 & 629 & 303 & 76 & 264 & 661 & 303 & 1 & 4 & 11 & 5 \\
\hline \begin{tabular}{|l} 
Agrio Fold Belt \\
Continuous Oil AU \\
\end{tabular} & 1.0 & Oil & 87 & 325 & 813 & 372 & 81 & 317 & 844 & 371 & 1 & 5 & 14 & 6 \\
\hline Total resources & & & 169 & 597 & 1,442 & 675 & 157 & 581 & $\mathbf{1 , 5 0 5}$ & 674 & 2 & 9 & 25 & 11 \\
\hline \begin{tabular}{|c|}
$\begin{array}{c}\text { Total undiscovered } \\
\text { continuous resources }\end{array}$ \\
\end{tabular} & & & 4,139 & 13,439 & 27,913 & 14,370 & 10,382 & 34,863 & 76,102 & 37,966 & 113 & 422 & 1,055 & 482 \\
\hline
\end{tabular}

\section{Neuquén Basin Province Assessment Team}

Christopher J. Schenk, Timothy R. Klett, Marilyn E. Tennyson,

Tracey J. Mercier, Janet K. Pitman, Stephanie B. Gaswirth, Thomas M. Finn, Michael E. Brownfield, Phuong A. Le, Heidi M. Leathers-Miller, and Kristen R. Marra

\section{For More Information}

Assessment results are available at the USGS Energy Resources Program website at https://energy.usgs.gov. 